\title{
Water-in-Oil Emulsions Prepared by Peptide-Silicone Hybrid Polymers as Active Interfacial Modifier: Effects of Silicone Oil Species on Dispersion Stability of Emulsions
}

\author{
Kenichi Sakai * , Satoshi lijima ${ }^{1}$, Ryosuke Ikeda', Takeshi Endo ${ }^{1}$, Takahiro Yamazaki ${ }^{2}$, \\ Yuji Yamashita ${ }^{2}$, Makoto Natsuisaka ${ }^{3}$, Hideki Sakai', Masahiko Abe $^{1 *}$ and \\ Kazutami Sakamoto, $2 *$ \\ ${ }^{1}$ Department of Pure and Applied Chemistry in Faculty of Science and Technology, and Research Institute for Science and Technology, Tokyo \\ University of Science, 2641 Yamazaki, Noda, Chiba 278-8510, Japan. \\ ${ }^{2}$ Faculty of Pharmacy, Chiba Institute of Science, 15-8 Shiomi-cho, Choshi, Chiba 288-0025, Japan. \\ ${ }^{3}$ Institute of Space and Astronautical Science (ISAS), Japan Aerospace Exploration Agency (JAXA), 2-1-1 Sengen, Tsukuba, Ibaraki 305-8505, \\ Japan.
}

\begin{abstract}
We have recently proposed a new general concept regarding amphiphilic materials that have been named as "active interfacial modifier (AIM)." In emulsion systems, an AIM is essentially insoluble in both water and organic solvents; however, it possesses moieties that are attracted to each of these immiscible liquid phases. Hence, an AIM practically stays just at the interface between the two phases and makes the resulting emulsion stable. In this study, the effects of silicone oil species on the dispersion stability of waterin-oil (W/O) emulsions in the presence of an AIM sample were evaluated in order to understand the destabilization mechanism in such emulsion systems. The AIM sample used in this study is an amphiphilic polymer consisting of a silicone backbone modified with hydrocarbon chains and hydrolyzed silk peptides. The Stokes equation predicts that the sedimentation velocity of water droplets dispersed in a continuous silicone oil phase simply depends on the expression $\left(\rho-\rho_{0}\right) / \eta$ assuming that the droplet size is constant (where $\rho$ is the density of the dispersed water phase, $\rho_{0}$ is the density of the continuous silicone oil phase, and $\eta$ is the viscosity of the oil phase). The experimental results shown in this paper are consistent with the Stokes prediction: i.e., in the low-viscous genuine or quasi-Newtonian fluid region, the dispersion stability increases in the following order: dodecamethylpentasiloxane (DPS) $<$ decamethylcyclopentasiloxane $\left(D_{5}\right) \leq$ dodecamethylcyclohexasiloxane $\left(\mathrm{D}_{6}\right)$. This order agrees well with the order obtained by using the expression $\left(\rho-\rho_{0}\right) / \eta$ as DPS $>D_{5}>D_{6}$. This indicates that our emulsion system experiences destabilization through sedimentation, but hardly any coalescence occurs owing to the presence of an additional third phase consisting of the AIM that stabilizes the silicone oil/water interface in the emulsions.
\end{abstract}

Key words: Active Interfacial Modifier (AIM), Emulsion, Coalescence, Rheology, The Stokes Equation

\section{INTRODUCTION}

When surfactants are adsorbed at gas/liquid, liquid/ liquid, or solid/liquid interfaces, a remarkable change in their interfacial properties occurs. For example, emulsions consist of liquid droplets dispersed in a continuous liquid phase (where the two different liquids are intrinsically immiscible with each other), and surfactants stabilize the emulsion droplets as a result of their adsorption at the liquid/liquid interface in the emulsions. In general, surfactants are distributed into the interface layer as a surface excess toward bulk concentration, and hence, the effective amount of surfactants required to stabilize the interface becomes significantly lower than the added amount in the system. In order to achieve a precise control over the interfacial properties with the lowest expense of energy and materials, the concentration of surfactants dissolved in continuous liquid phases must be minimized. Ideally, surfactants are required to be only present between the two phases in the emulsions.

From this point of view, we have recently proposed a

\footnotetext{
*Correspondence to: Kenichi Sakai, Department of Pure and Applied Chemistry in Faculty of Science and Technology, and Research Institute for Science and Technology, Tokyo University of Science, 2641 Yamazaki, Noda, Chiba 278-8510, Japan.

E-mail: k-sakai@rs.noda.tus.ac.jp (KS); abemasa@rs.noda.tus.ac.jp (MA); kasakamoto@ cis.ac.jp (KS)

Accepted February 27, 2013 (received for review January 10, 2013)

Journal of Oleo Science ISSN 1345-8957 print / ISSN 1347-3352 online

http://www.jstage.jst.go.jp/browse/jos/ http://mc.manusriptcentral.com/jjocs
} 
new general concept regarding the amphiphilic materials that have been named as "active interfacial modifier (AIM)" ${ }^{1)}$. In emulsion systems, AIM is intrinsically insoluble in both water and organic solvents, but possesses moieties that are attracted to each of these immiscible liquid phases. Hence, an AIM practically stays just at the interface, thereby contributing to the formation of a stable emulsion. Several emulsion systems are known to somehow adapt to such requirements, where the "emulsifiers" are localized at the oil/water interface and act as an independent third phase or layer. Hence, the AIM concept may be applicable to such systems as (i) "Pickering emulsion" stabilized with solid particles adsorbed at the interface ${ }^{2)}$, (ii) "three-phase emulsion" stabilized with a phospholipid bilayer $^{3)}$, (iii) "liquid crystal emulsion" ${ }^{4)}$, and (iv) "cubosomes" or "isasomes" stabilized with triblock copolymers ${ }^{5)}$.

In the previous work ${ }^{1}$, we have employed an amphiphilic peptide-silicone hybrid polymer as an example for the AIM concept. This hybrid material consists of silicone backbones grafted with hydrocarbon chains and hydrolyzed silk peptides. Hereafter, this material is abbreviated as "AIMFN" while the term "AIM" is used as the general name for such amphiphiles. The key property of AIM-FN is that it is insoluble in both water and most of the organic solvents but gets attracted to these solvents due to its amphiphilic nature. The addition of AIM-FN to a mixture of water and silicone oil (decamethylcyclopentasiloxane, $D_{5}$ ) gives stable water-in-oil $(\mathrm{W} / \mathrm{O})$ emulsions $($ droplet diameter $=1-4 \mu \mathrm{m})$ even by relatively gentle vortex mixing for a wide variety of ratios of the three components. The prepared W/O emulsions are nearly genuine or quasi-Newtonian fluid with low viscosities (the water contents range from 0 to $36 \mathrm{wt} \%$ for a given weight ratio of AIM-FN/D $\left.D_{5}=6: 4\right)$. These results suggest that AIM-FN stays as an independent third phase (with a significant thickness) between the two liquid phases.

On the basis of the experimental results reported in the previous paper ${ }^{1)}$, we assume that the emulsion system experiences destabilization due to sedimentation process, but hardly any coalescence occurs owing to the presence of the AIM-FN phase localized between the dispersed water and continuous $\mathrm{D}_{5}$ phases. The sedimentation velocity $(v)$ is described by the Stokes equation ${ }^{6)}$ as follows:

$$
v=\frac{2 r^{2}\left(\rho-\rho_{0}\right) g}{9 \eta},
$$

where $r$ is the radius of emulsion droplets, $\rho$ is the density of dispersed phase (water), $\rho_{0}$ is the density of continuous phase (silicone oil), $\eta$ is the viscosity of continuous phase (silicone oil), and $g$ is the gravitational acceleration. Therefore, the use of various silicone oils enables us to determine the destabilization mechanism of the W/O emulsion systems. Hereafter, the effects of silicone oil species on the dispersion stability of the AIM-FN emulsion system will be discussed. The main aim of this study is to provide the experimental evidence for the destabilization mechanism operating in such emulsion systems. This may be useful in understanding the similarities and/or differences between our AIM system and other emulsion systems such as "Pickering emulsion," "three-phase emulsion," and "liquid crystal emulsion."

\section{EXPERIMENTAL}

\subsection{Materials}

A hydrolyzed silk PG-propyl methylsilanediol crosspolymer sample was used as the AIM in the current study. This polymer sample was the main component of Seiwa Kasei PROTESIL $^{\circledR}$ FN and was synthesized as reported elsewhere by coupling of silk peptides with the reactive silicone monomers ${ }^{7-9}$. This polymer consists of silicone backbones grafted with hydrocarbon $\left(n-\mathrm{C}_{8} \mathrm{H}_{17}\right)$ chains and hydrolyzed silk peptides. This polymer sample with a paste-like appearance is intrinsically insoluble in water, and thus, a twophase separation occurs when AIM-FN is added to water. Similarly, AIM-FN is also intrinsically insoluble in silicone oils, but it could be dispersed in these oils by vortex mixing. We have examined the organic conceptual (OC) diagram $^{10)}$ of this AIM material by plotting the calculated inorganic value (IV) and organic value (OV) by using the values given for each chemical moiety. The value of IV/OV ratio of AIM-FN is calculated to be ca. 5000/8700 (IV/OV = 0.57 ), based on its monomer composition. The IV/OV ratio corresponds to the hydrophilic-lipophilic balance (HLB) of amphiphilic materials and the ratio calculated for AIM-FN is within the range of traditional polyoxyethylene-type emulsifiers with an HLB value around 3 to 6 , which is suitable for forming a W/O emulsion.

The silicone oils employed in this study were as follows: $\mathrm{D}_{5}$, dodecamethylcyclohexasiloxane $\left(\mathrm{D}_{6}\right)$, and dodecamethylpentasiloxane (DPS). These silicone oils were purchased from Dow Corning Toray and used as received. Chemical structures and physicochemical properties of these silicone oils are shown in Fig. 1 and Table 1. The water used in this study was filtered with a Millipore membrane filter $(0.22$ $\mu \mathrm{m}$ in pore size) after deionization with a Barnstead NANO pure diamond UV system.
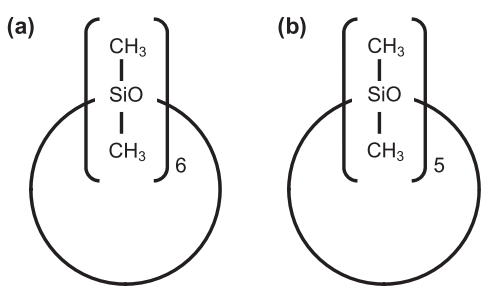

(c)

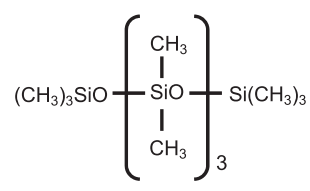

Fig. 1 Chemical structures of silicone oils used in this study: (a) $\mathrm{D}_{6}$, (b) $\mathrm{D}_{5}$, and (c) DPS. 
Table 1 Physicochemical properties of silicone oils used in this study.

\begin{tabular}{cccc}
\hline & Viscosity $\eta\left(\mathrm{mPa} \mathrm{s}^{\mathrm{a})}\right.$ & Density $\rho_{0}\left(\mathrm{~g} \mathrm{~cm}^{-3}\right)^{\mathrm{a})}$ & $\left(\rho-\rho_{0}\right) / \eta\left(\mathrm{s} \mathrm{m}^{-2}\right)$ \\
\hline $\mathrm{D}_{6}$ & 6.5 & 0.96 & $6.1 \times 10^{3}$ \\
$\mathrm{D}_{5}$ & 3.6 & 0.95 & $1.4 \times 10^{4}$ \\
DPS & 1.7 & 0.87 & $7.5 \times 10^{4}$ \\
\hline
\end{tabular}

a) These values are seen in the material safety data sheet(MSDS) provided by the supplier.

\subsection{Emulsification}

In the first step, the silicone oils were added to AIM-FN in a glass test tube and the AIM-FN/silicone oil system was heated for $3 \mathrm{~min}$ in a hot water bath set at $70^{\circ} \mathrm{C}$. Then, the components were mixed together by using a vortex mixer for $3 \mathrm{~min}$. In the second step, water was added into the AIM-FN/silicone oil dispersion system and the water/ AIM-FN/silicone oil system was again heated for 3 min in a hot water bath set at $70^{\circ} \mathrm{C}$. Final mixing of the emulsion system was done by using the vortex mixer for $3 \mathrm{~min}$.

\subsection{Measurements}

Optical microscope observations were made by using an Olympus IMT-2 microscope. A small amount of emulsion sample was placed in the hollow (0.5 mm depth) of a glass slide and covered with a cover glass. Shear viscosity measurements were performed using a TA Instruments AR-G2 rheometer with the cone-plate geometry (cone angle $=2^{\circ}$, diameter $=40 \mathrm{~mm})$. All measurements reported herein were performed at a constant temperature of $25^{\circ} \mathrm{C}$.

\section{RESULTS AND DISCUSSION}

\subsection{Rheology}

As mentioned in the introduction, we have already characterized the viscosity of the water-in- $\mathrm{D}_{5}$ emulsion system stabilized by AIM-FN ${ }^{1)}$. In this paper, we focus on the effects of silicone oil species on the viscosity in the emulsion systems. Figure 2 shows the static viscosity data obtained for the three emulsion systems: (a) $D_{6}$, (b) $D_{5}$, and (c) DPS. The composition ratio of AIM-FN/silicone oil was fixed at 6:4 (in wt\%) and water content was varied from 0 to $50 \mathrm{wt} \%$. These measurements were performed immediately after the preparation of the emulsion samples. For all of the emulsion systems investigated in this study, it was
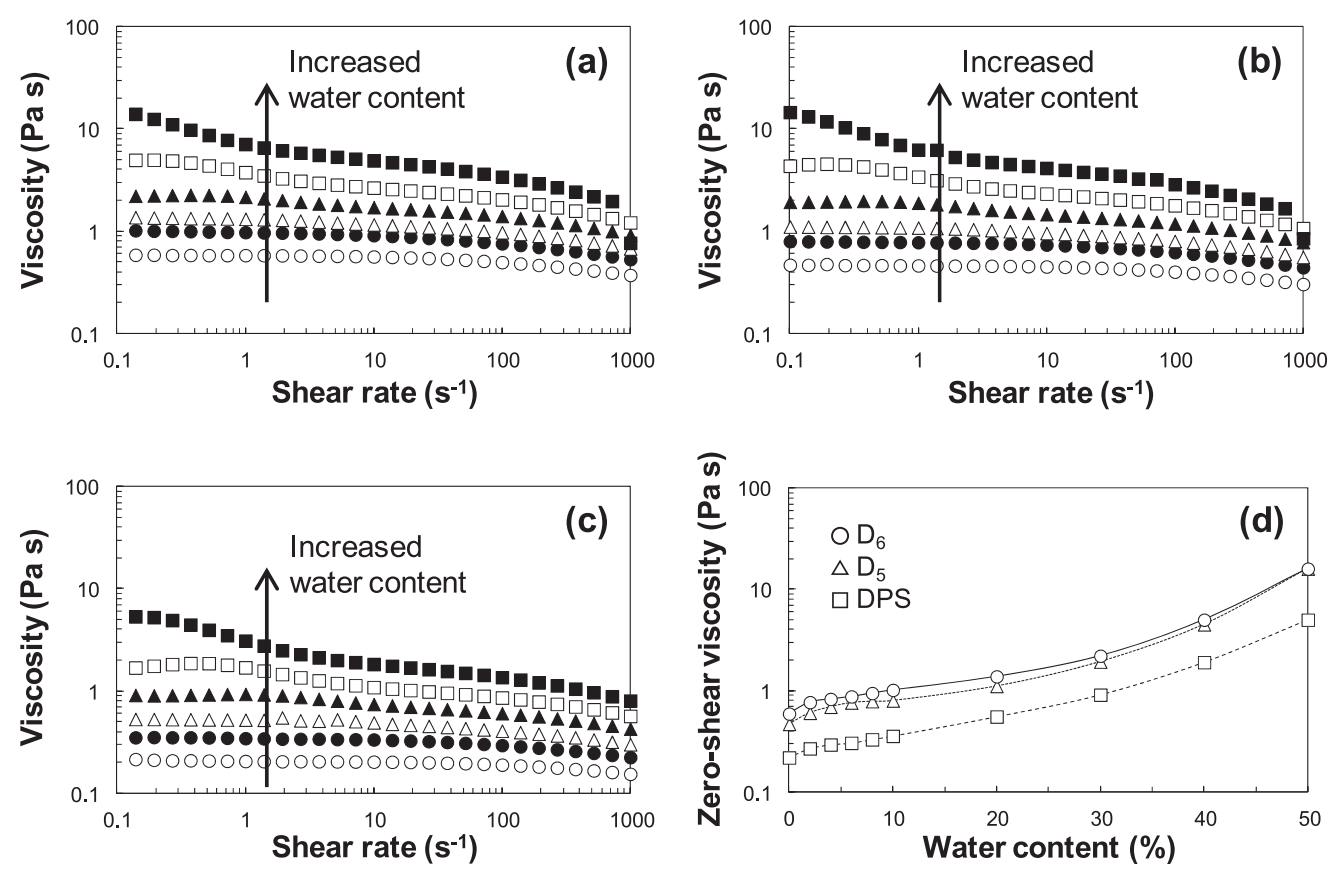

Fig. 2 Static viscosity data obtained for the three emulsion systems: (a) $\mathrm{D}_{6}$, (b) $\mathrm{D}_{5}$, and (c) DPS. The composition ratio of AIM-FN/silicone oil was fixed at $6: 4$ (in wt $\%)$ and the water content was varied from 0 to $50 \mathrm{wt} \%(0,10,20$, 30,40 , and $50 \mathrm{wt} \%$ ). These measurements were performed immediately after the preparation of the emulsion samples. The zero-shear viscosity data are plotted in (d) as a function of water content. The zero-shear viscosity was determined by extrapolating the viscosity in the resulting shear rate curve to zero-shear rate. 
observed that an increased water content results in an increased viscosity at a given shear rate. This is clearly seen when plotting the zero-shear viscosity data as a function of water content, as shown in Fig. 2 (d). One can also see in Fig. 2 (a) - (c) that independent of the silicone oil species, a nearly genuine or quasi-Newtonian fluid with low viscosity is obtained at low water contents (less than $30 \mathrm{wt} \%$ ), whereas non-Newtonian shear thinning behavior is observed at high water contents (greater than $30 \mathrm{wt} \%$ ). We have suggested in our previous paper that the emulsion droplets shear at the interface between the continuous silicone oil phase and the shell-like AIM-FN phase, thereby encapsulating the inner water phase ${ }^{1)}$. It seems likely that in this situation, the interactions between the water droplets have a significant effect on the emulsion viscosity: i.e., the greater viscosity (and the non-Newtonian shear thinning behavior) observed at high water contents results from a network structure in the emulsion droplets, whereas the low-viscous Newtonian fluid obtained at low water contents suggests that no significant interaction between the emulsion droplets takes place ${ }^{1)}$.

As is seen in Fig. 2(d), the emulsion viscosity depends on the silicone oil species: DPS $<\mathrm{D}_{5} \leq \mathrm{D}_{6}$ (at a given water content). This order is consistent with the order of silicone oil viscosity listed in Table 1. This suggests that the emulsion viscosity is largely affected not only by the water content but also by the viscosity of continuous silicone oil phase, as expected.

\subsection{Dispersion stability}

The dispersion stability of emulsions was assessed on the basis of visual and optical microscope observation results. As a typical example, we selected a composition of AIM-FN/silicone oil/water $=54: 36: 10$ (in wt\%), where lowviscous Newtonian fluids are obtained for all of the three emulsion systems (Fig. 2). The emulsion samples prepared

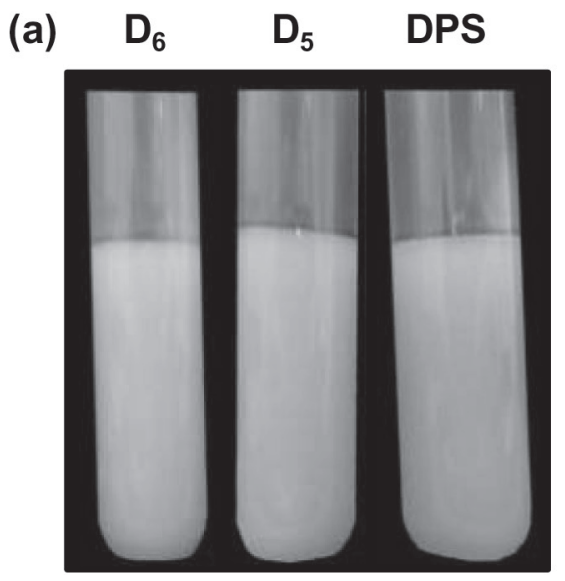

for this study were very stable over a month, and hence we performed an acceleration test by using a Kokusan H-28F centrifuge (arm length $=0.14 \mathrm{~m}$, rotation speed $=3500$ $\mathrm{rpm}$ ). Figure 3 shows the visual appearance of the emulsion samples obtained before and after the centrifugation for $6 \mathrm{~h}$. For all three emulsion samples, the centrifugation results in a phase separation of silicone oils from the emulsion phase. The volume percent of the oil phase against the total sample volume was estimated from these pictures. The results are shown in Fig. 4. The volume percent of the oil phase increases with increasing centrifugation time. More importantly, the volume percent of the oil phase is larger for the DPS system than those for the $\mathrm{D}_{5}$ and $\mathrm{D}_{6}$ systems. Again, this is consistent with the order of the viscosity of the continuous silicone oil phase.

We determined the emulsion droplet size by optical mi-

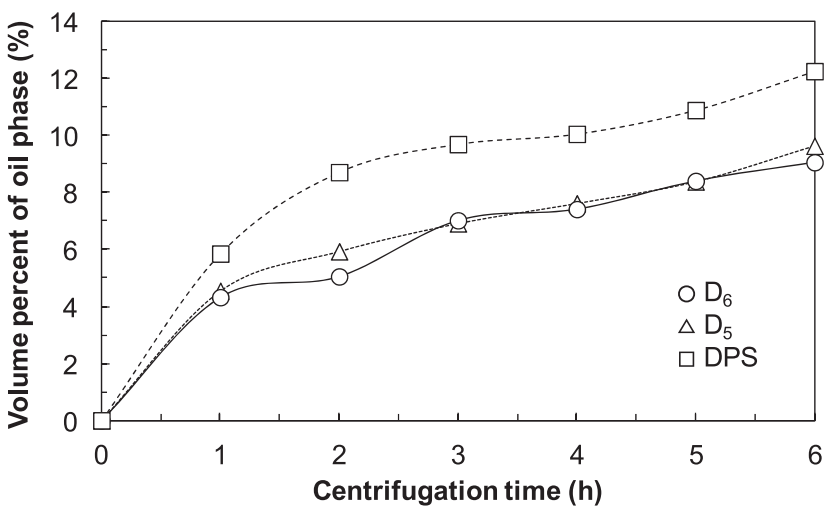

Fig. 4 Volume percent of silicone oil phase as a function of centrifugation time. These data were obtained on the basis of visual observation results, where the emulsion samples were prepared at a fixed composition of AIM-FN/silicone oil/water $=54: 36: 10$ in $\mathrm{wt} \%$.

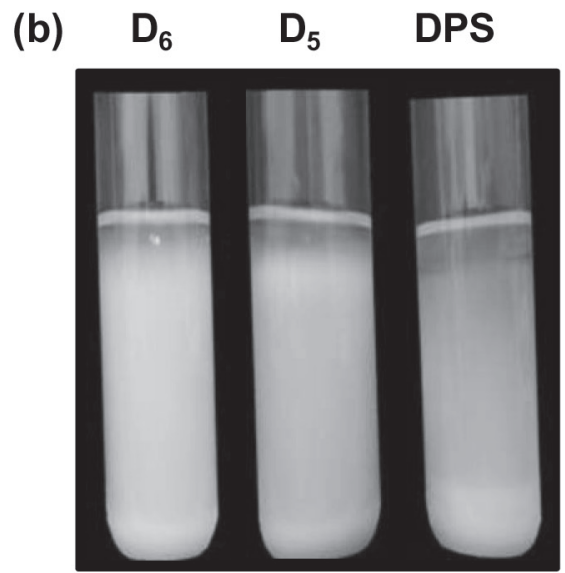

Fig. 3 Visual observation results of emulsion samples: (a) before and (b) after centrifugation for $6 \mathrm{~h}$. The emulsion samples were prepared at a fixed composition of AIM-FN/silicone oil $/$ water $=54: 36: 10 \mathrm{in} \mathrm{wt} \%$. 
croscope observations. Figure 5 shows the images obtained in all the three emulsion systems. The size distribution was estimated by measuring the diameter of randomly placed 100 droplets shown in these images (or additional images focusing on different microscope fields). On the basis of these data, we suggest that the oil species do not have a significant effect on the droplet size and their distribution (before centrifugation). Equation 1 predicts the ease of sedimentation process as a function of $\left(\rho-\rho_{0}\right) / \eta$ under the assumption that the droplet size is constant for all the

(a)

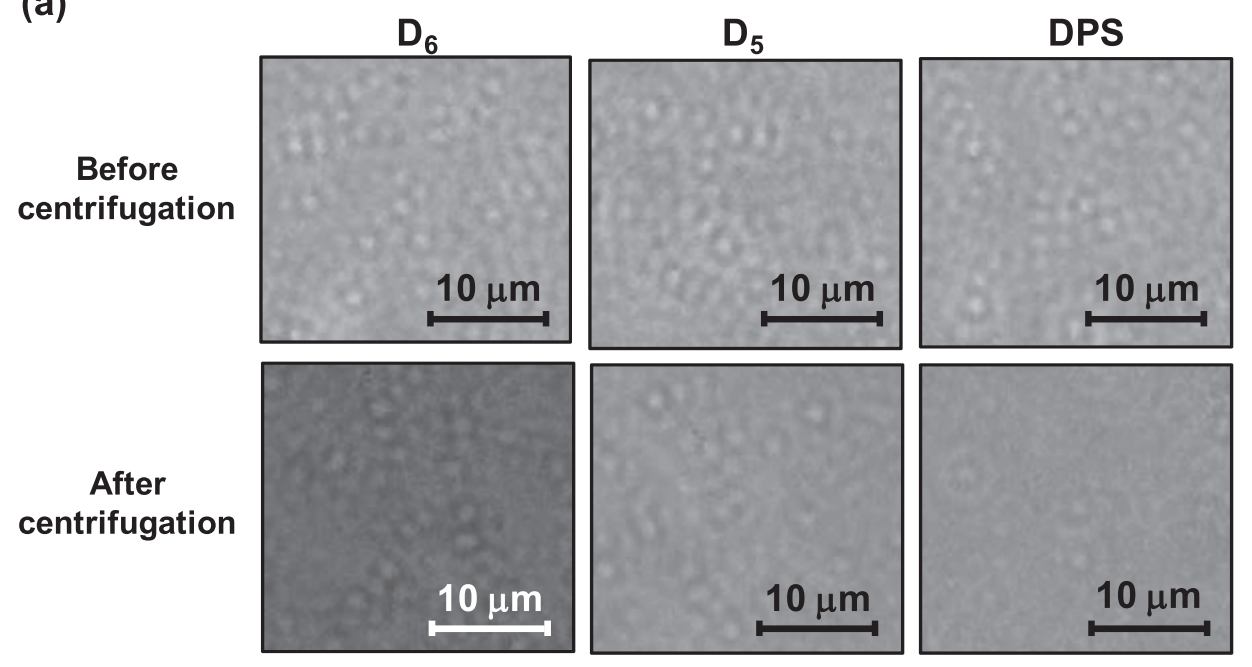

(b)
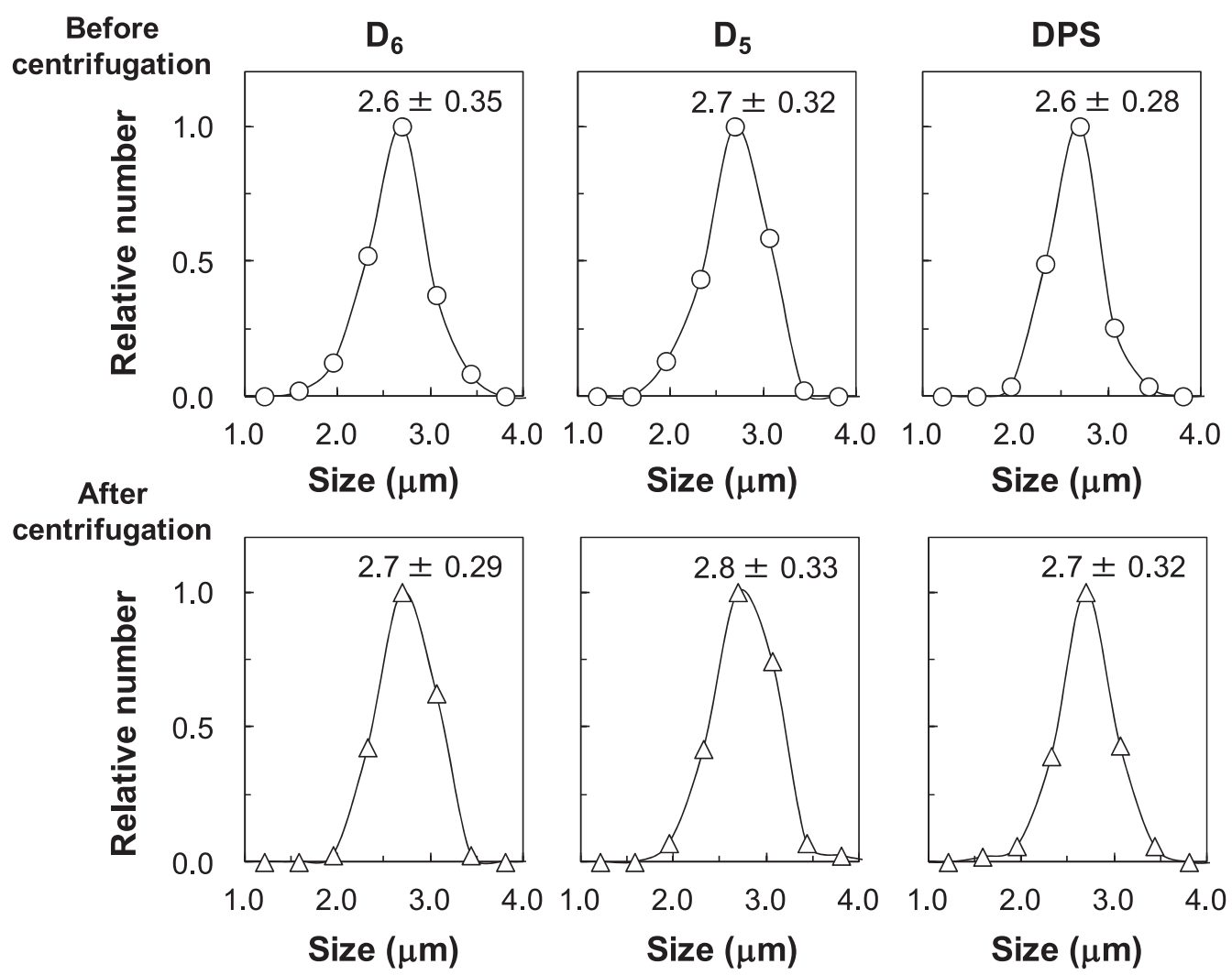

Fig. 5 (a) Optical microscope images of the emulsion samples prepared at a fixed composition of AIM-FN/silicone oil/ water $=54: 36: 10 \mathrm{in} \mathrm{wt} \%$. These measurements were performed both before and after the centrifugation for $6 \mathrm{~h}$. (b) The corresponding size distribution data. 
three emulsion systems. This assumption is in agreement with the experimental results in this study. Therefore, we can measure the dispersion stability by using the expres$\operatorname{sion}\left(\rho-\rho_{0}\right) / \eta$. These results strongly indicate that the sedimentation velocity of the DPS system is higher than those of the $D_{5}$ and $D_{6}$ systems, since the DPS system has the largest $\left(\rho-\rho_{0}\right) / \eta$ value, as shown in Table 1.

We observed that the centrifugation experiments led to not only the separation of the silicone oils at the top of the samples but also the sedimentation as a milky fluid at the bottom of the samples. We assume that the milky fluid obtained in this study results from an excess amount of AIM-FN used in the process. It is not clear whether the excess amount of AIM-FN is originally dispersed in the silicone oils (without encapsulating the water droplets) or whether the centrifugation tears down the AIM-FN phase from the dispersed water droplets. Nevertheless, on the basis of visual and optical microscope observations, it can be concluded that(i) the centrifugation does not induce a significant change in the emulsion droplet size (in the "middle" emulsion phase), as shown in Fig. 5; and(ii) gentle hand-mixing allows for re-dispersion of the phaseseparated emulsion samples without a change in the droplet size. This supports our hypothesis that the emulsion systems used in this study experience destabilization not through coalescence but mainly via a sedimentation process.

It would be interesting to find the possible reasons why hardly any coalescence occurs in our emulsion systems. It is generally accepted that coalescence occurs as a result of the destruction of interfacial layers in between two neighboring emulsion droplets. Therefore, highly stable non-coalescent emulsion systems are obtained when sufficient physicochemical or mechanical strength (in other words, sufficient viscoelastic nature) is provided to the interfacial layer. For example, in liquid crystal emulsion systems, the liquid crystals surrounding the oil droplets yield a very stable non-coalescent oil-in-water emulsion system by acting as an independent third phase or layer ${ }^{4)}$. In this study, we assume that AIM-FN acting as a third phase plays a similar role as the liquid crystals. The highly non-coalescent nature of our emulsion systems may result from the significant thickness of the AIM-FN phase that helps to reduce the oil/water interfacial energy level down to zero by virtue of its nature. Unfortunately, we have no direct experimental evidence supporting this claim at present. In future, more detailed physicochemical studies will be performed by interfacial tensiometry and/or interfacial rheology techniques to prove this claim.

\subsection{Visual observation of "millimeter-sized" emulsion droplets}

In this section, we present the preliminary results regarding the preparation of "millimeter-sized" emulsion droplets using the AIM-FN/D $\mathrm{D}_{5} /$ water system. The giant emulsion droplets were prepared as follows. The commercially available PROTESIL ${ }^{\circledR} \mathrm{FN}$ (whose main component is AIM-FN) $(1 \mathrm{~g})$ was dispersed in $\mathrm{D}_{5}(9 \mathrm{~g})$, and then, pure water was added dropwise to the mixture under a constant flow rate of ca. $48 \mathrm{~cm}^{3} / \mathrm{h}$. Figure 6 shows a snapshot of such giant droplets. In the absence of AIM-FN, the coalescence of water droplets takes place very quickly after the dropwise addition, and hence, "water pools" are formed at the bottom of the glass vial. In contrast, in the presence of AIM-FN, "millimeter-sized" water droplets retain their structures at the bottom of the vial (see Fig. 6). The particle size may be controlled by changing the size of the nozzle and/or the flow rate. Interestingly, the increased number of the giant droplets in the vial leads to a stack of droplets with a closely packed order. This is a good piece of evidence that AIM-FN forms an independent third phase around the water droplets and stabilizes the $\mathrm{D}_{5} /$ water interface against the coalescence process. We also note here that an opaque emulsion with a particle size of less than $300 \mathrm{~nm}$ can be formed at appropriate compositions by vortex or other mechanical mixing (data are not shown here because the topic is beyond the scope of this study). Therefore, it is possible to prepare emulsion droplets with a wide range of diameters (from nanometer to millimeter sizes) by using AIM-FN (or other such amphiphilic materials).

\section{CONCLUSIONS}

We have studied the effects of silicone oil species on the dispersion stability of the W/O emulsions stabilized by AIM-FN. The Stokes equation predicts that the sedimentation velocity of the water droplets dispersed in a continuous oil phase simply depends on the expression $\left(\rho-\rho_{0}\right) / \eta$ under an assumption that the droplet size is constant. The

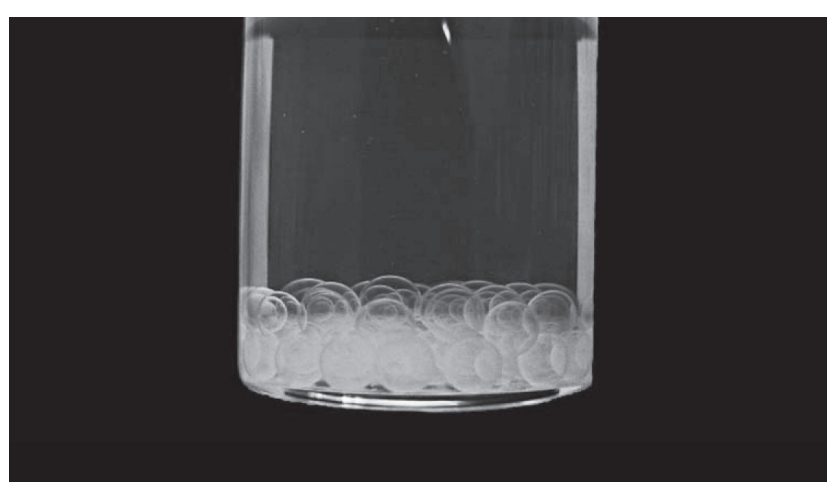

Fig. 6 Visual appearance of "millimeter-sized" emulsion droplets. This snapshot was taken immediately after the dropwise addition of pure water into the mixture of PROTESIL ${ }^{\circledR}$ FN with $\mathrm{D}_{5}$. 
experimental results shown in this paper are consistent with the Stokes prediction; i.e., in the low-viscous quasiNewtonian fluid region, the dispersion stability is increased as DPS $<\mathrm{D}_{5} \leq \mathrm{D}_{6}$, and this order is in good agreement with the order of $\left(\rho-\rho_{0}\right) / \eta$ values, which is DPS $>D_{5}>D_{6}$. These physicochemical data and the experimental results leading to the additional "millimeter-sized" giant emulsion droplets indicate that AIM-FN forms an independent third phase around the water droplets and stabilizes the silicone oil/ water interface against the coalescence process. This means that our emulsion system experiences destabilization via a sedimentation process, but hardly any coalescence occurs owing to the presence of the AIM-FN layer acting as the third phase in the emulsions.

As mentioned in the introduction, the AIM is a general concept for treating immiscible liquid/liquid interfaces using a material that practically stays just at the interface of the emulsions. Hence, the AIM may be applicable in the case of not only W/O emulsions prepared by AIM-FN but also "Pickering emulsion," "three-phase emulsion," "liquid crystal emulsion," etc. We hope that the findings reported in this paper will provide a new insight regarding the destabilization mechanism of such emulsion systems.

\section{ACKNOWLEDGEMENTS}

We thank Seiwa Kasei for providing the hybrid amphiphilic material for us. A part of this work was supported by Japan Aerospace Exploration Agency (JAXA).

\section{REFERENCES}

1) Sakai, K.; Ikeda, R.; Sharma, S. C.; Shrestha, R. G.;
Ohtani, N.; Yoshioka, M.; Sakai, H.; Abe, M.; Sakamoto, K. Active interfacial modifier (AIM): Stabilization mechanism of water in silicone oil emulsions by peptide-silicone hybrid polymers. Langmuir 26, 53495354 (2010).

2) Pickering, S. U. Emulsions. J. Chem. Soc. Trans. 91, 2001-2021 (1907).

3) Tajima, K.; Imai, Y.; Tsutsui, T. Structure of threephase emulsion stabilized with phospholipid bilayerassembly and its stability. J. Oleo. Sci. 51, 285-296 (2002).

4) Suzuki, T.; Takei, H.; Yamazaki, S. Formation of fine three-phase emulsions by the liquid crystal emulsification method with arginine $\beta$-branched monoalkyl phosphate J. Colloid Interface Sci. 129, 491-500 (1989).

5) Spicer, P. T.; Hayden, K. L.; Lynch, M. L.; OforiBoateng, A.; Burns, J. L. Novel process for producing cubic liquid crystalline nanoparticles (cubosomes). Langmuir 17, 5748-5756 (2001).

6) Becher, P. Emulsions: Theory and Practice; Maruzen: Tokyo, Chapter 5(1960).

7) Koyanagi, A.; Goto, N.; Daikai, S.; Uchida, S.; Hayashi, N.; Yoshioka, M. Novel multi-functional hybrid polymer. J. Soc. Cosmet. Chem. Jpn. 41, 269-274 (2007).

8) Koyanagi, A. Hydrolyzed protein derivatives accompanied with synergy by silylation or siliconizing. $J$. Cosmet. Sci. 58, 435-441 (2007).

9) Daikai, S. Development and application of a novel amino acid derivative and a novel peptide derivative for hair care. Fragrance J. 35, 49-54 (2007).

10) Koda, Y.; Sato, S.; Honma, Y. Organic Conceptual Diagram, Basis and Application; Sankyo: Tokyo (1984). 